\title{
Missions en faveur de la protection des Langues des Signes en danger dans la région de l'Océan Indien: la Langue des Signes Mauricienne (République de Maurice) et de la Langue des Signes Seychelloise (République des Seychelles), en lien avec le Pôle LSF de l'INJS de Paris
}

\author{
Missão em defesa da proteção de Línguas de Sinais em risco \\ na região do Oceano Índico: a Língua de Sinais Maurícias \\ (República de Maurício) e a Língua de Sinais de Seychelles \\ (República de Seychelles), em conexão com o Polo LSF do \\ INJS de Paris
}

\author{
Monique GENDROT \\ interprète Français/LSF \\ Alain GEBERT \\ Pôle LSF de l'INJS de Paris
}

\begin{abstract}
RÉSUMÉ: Le 4 Mai 2006, la publication du premier tome du Dictionnaire de la Langue des Signes Mauricienne (MSL) était officiellement lancée par son Hon. S.Bappoo, Ministre de la Sécurité Sociale à l'Ile Maurice. Le 17 Mars 2007, le premier Journal Télévisé Hebdomadaire en MSL interprété par des présentatrices Sourdes voyait le jour sur les chaînes nationales mauriciennes de la Mauritius Broadcasting Corporation. Ces deux évènements majeurs ont participé à la reconnaissance officielle de la MSL par le gouvernement mauricien d'abord, puis par l'ONU. Ils ont été réalisés dans le cadre d'une convention signée entre le gouvernement mauricien, l'INJS de Paris, l'Ambassade de France à Maurice et l'Université de Düsseldorf pour le Développement de la Langue des Signes Mauricienne et ce, pour une durée de 3 ans, de 2005 à 2007. Ce projet œuvrait en conformité avec les Règles des Nations Unies portant sur l'Egalisation des Chances pour les Personnes Handicapées.
\end{abstract}

MOTS-CLÉ: Dictionnaire de langue des signes. Langue en danger. Langue des signes mauricienne et seychelloise. Accessibilité TV. Schématisation.

RESUMO: Em 4 de maio 2006, a publicação do primeiro tomo do Dicionário de Língua de Sinais Mauriciana (MSL) foi oficialmente lançada por son Hon. S. Bappoo, Ministro da Segurança Social das Ilhas Maurícias. 17 de março de 2007, nascia, nos canais nacionais maurícios da Mauritius Broadcasting Corporation, o primeiro Jornal Televisionado Semanal em MSL interpretado por apresentadoras Surdas. Esses acontecimentos maiores participaram do reconhecimento oficial da MSL pelo governo maurício primeiramente, depois pela ONU. Essas ações foram realizadas no âmbito de uma convenção assinada entre o governo maurício, INJS de Paris, Embaixada da França nas Ilhas Maurício e Universidade de Düsseldorf para o Desenvolvimento da Língua de Sinais Maurício, por uma duração de 3 anos, de 2005 a 2007. Esse projeto trabalhava em conformidade com as Regras das Nações Unidas sobre a Igualdade de Oportunidades para as Pessoas Deficientes.

PALAVRAS-CHAVE: Dicionário de língua de sinais. Língua em risco. Língua de sinais maurício (MSL). Acessibilidade na TV. Esquematização. 


\section{Introduction}

A l'occasion d'un séjour d'été en 2002, Mr Alain Gébert, Sourd d'origine mauricienne et professeur de LSF à l'INJS de Paris prend la mesure de la mise en danger de la Langue des Signes Mauricienne, minoritaire et émergente, et de la culture qu'elle sous-tend. En effet, sous l'influence grandissante de la Langue des Signes Américaine (ASL), elle est menacée de disparition. Avec l'appui de la Présidente de l'ONG locale, la Society for the Welfare for the Deaf, Alain démarche alors le gouvernement mauricien afin de l'alerter, puis demande à l'Institut de bien vouloir s'inscrire dans un partenariat avec ce dernier. Il se retrouve ainsi responsable du projet tout en réalisant le premier tome du Dictionnaire de MSL avec Dany Adone, linguiste de l'Université de Düsseldorf et d'origine mauricienne. Cette dernière en rédige la partie grammaticale. Alain s'occupe de renseigner la langue en collectant ses signes. Il réalise la maquette finale du premier tome du Dictionnaire de MSL avant d'en confier la publication à une maison d'édition mauricienne.

\section{Le premier tome du dictionnaire de MSL : recherche et methodologie}

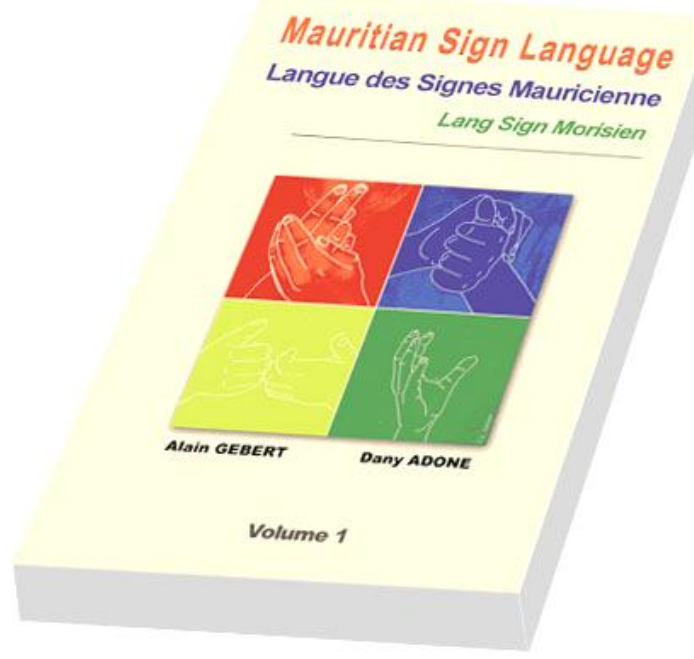

De 2002 à 2005, à raison de plusieurs missions annuelles programmées sur ses périodes de congés, Alain rassemble les Sourds de la communauté locale, se charge de recueillir des corpus, de les analyser, d'en extraire des signes, de les faire valider par les Sourds eux-mêmes avant de les illustrer pour la publication.

Durant ces trois années sa méthodologie évolue. Si au départ il utilise des images européennes afin d'obtenir des locuteurs locaux le lexique afférent en MSL, il les abandonne bien vite au profit d'images ou de photos tirées du contexte culturel mauricien. 
Figure 1: Extraction des signes d'après des images « européennes »
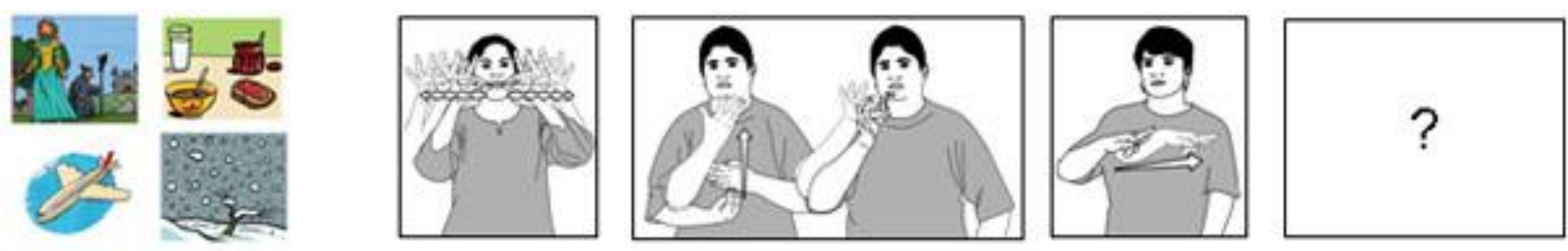

Source: GEBERT, A., 2005.

Figure 2: Extraction des signes d'après des photos issues du contexte mauricien
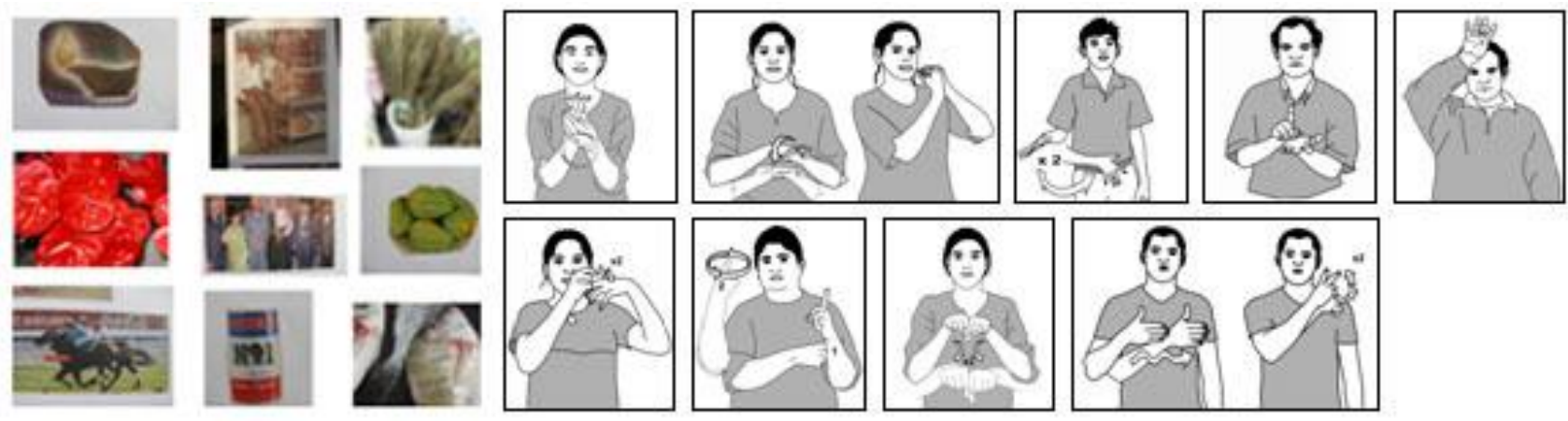

Source: Ibidem, 2005.

Toutefois, au hasard d'un signe à renseigner, celui de [POISSON CAPITAINE], la méthode employée s'avère encore insatisfaisante. En effet, le signe composé obtenu est différent de celui énoncé dans une production spontanée. Alain décide alors de renseigner la langue exclusivement à partir de corpus vidéo enregistrés en contexte auprès de locuteurs locaux.

Figure 3: Extraction des signes d'après des photos issues du contexte mauricien pour le signe [POISSON CAPITAINE]
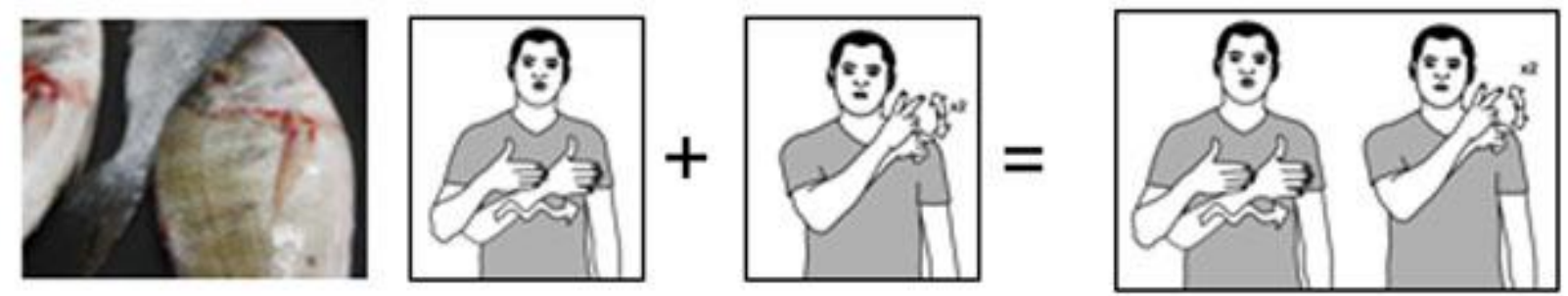

Source: Ibidem, 2005.

Figure 4: Extraction des signes d'après discours vidéo enregistré pour le signe [POISSON CAPITAINE] 

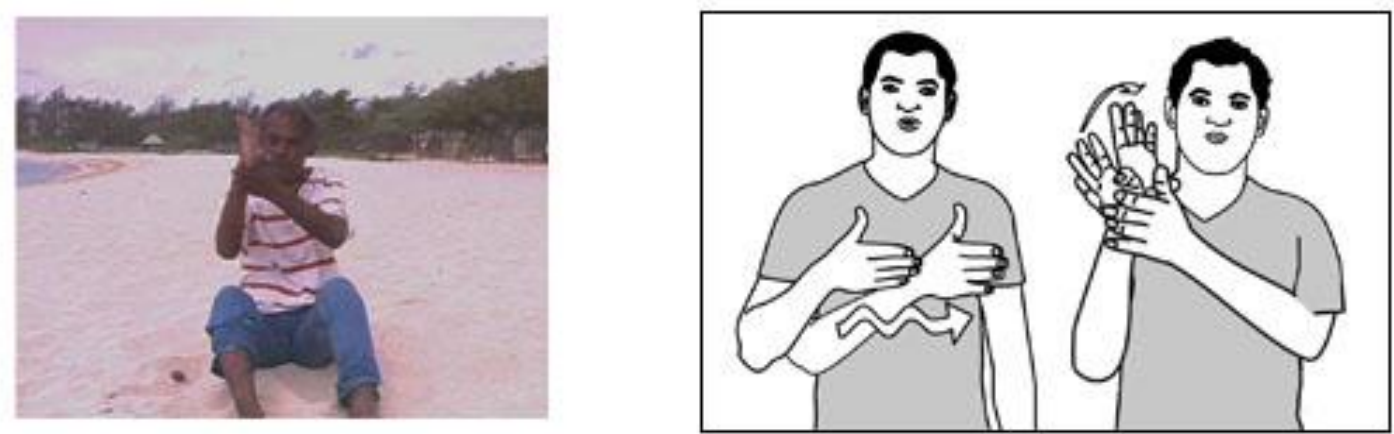

Source: Ibidem, 2005.

Si cette méthode demande davantage de temps, elle n'en demeure pas moins celle qui renseigne la langue au plus près de son authenticité via son expression spontanée. Ainsi revue et corrigée, elle permet d'établir un lien entre la gestualité mauricienne et certains signes de la MSL qui y puisent ses racines. En effet, l'île a traversé plusieurs périodes d'occupation. Elle a connu l'apport d'esclaves venus d'Afrique, de travailleurs engagés originaires de l'Inde. Ce brassage des cultures et la diversité des langues en présence ont laissé émerger une gestualité naturelle entre les populations mises ainsi en contact, y compris parmi la population sourde qui s'en est emparée. Des signes tels [se sauver] ou [punir] en sont issus.

Cette méthode dont l'avantage et le mérite est de renseigner la langue à sa source, dans son contexte culturel reste la seule utilisée encore à ce jour.

\section{Le Journal Televise hebdomadaire en MSL : son concept, sa methodologie}

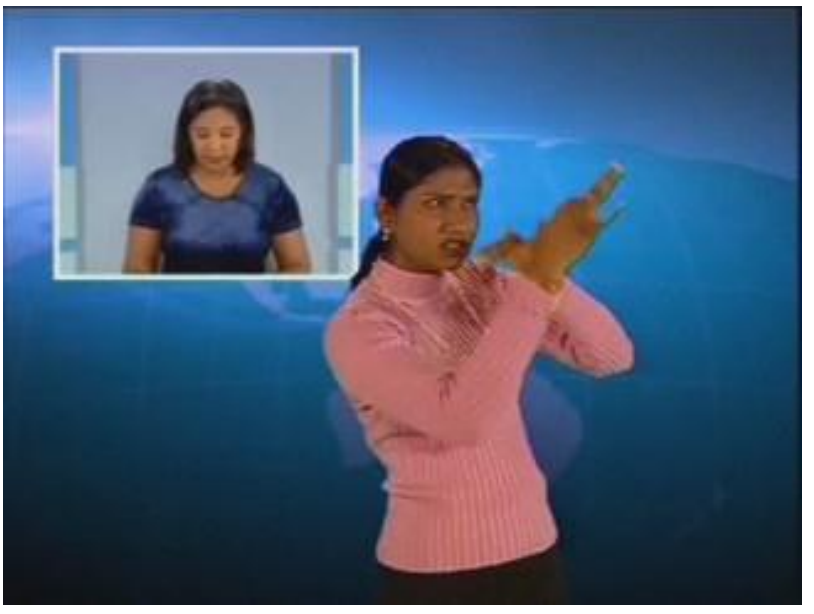

En Novembre 2006, soit 6 mois après la publication du Premier Tome du Dictionnaire et à la demande $\mathrm{du}$ gouvernement mauricien, Alain charge Monique Gendrot, interprète LSF/Français à mi-temps à l'INJS de Paris, de concevoir un bulletin d'informations télévisé sur les chaînes 
nationales mauriciennes. En effet depuis 1991, cette dernière traduit un journal d'informations destiné aux sourds et aux malentendants sur une chaîne de France Télévisions.

\subsection{Son concept}

En l'absence d'interprètes professionnels sur le terrain et au vu de la parution récente du premier tome du dictionnaire, Monique détermine les choix suivants :

- former des présentatrices Sourdes, locutrices de leur langue, préalablement instruites à son enseignement et à sa linguistique par les équipes de l'INSHEA (4 missions entre 2006 et 2007) et l'Université Paris 8 (1 mission en 2006 puis 1 en 2007).

- les placer au premier plan dans l'écran de télévision, la journaliste figurant en incruste en haut et à gauche de ce dernier. A l'inverse du modèle français, elle favorise ainsi l'accès au message en MSL pour le public sourd et entendant.

\subsection{Ses outils}

En dépit d'un programme d'alphabétisation à l'Alliance Française d'une durée de 6 mois, les présentatrices ne maitrisent pas suffisamment le français pour aborder seules les textes des brèves d'information. Par conséquent, nous constituons une équipe mixte composée de 4 enseignantes Sourdes de MSL et de 3 enseignants entendants (apprentis-interprètes). Ces derniers ne maitrisent pas encore la MSL dont le lexique vient tout juste d'être renseigné. Tous travaillent au sein de la même école pour enfants sourds à Beau-Bassin.

Ils préparent le Journal Télévisé à partir des textes et des reportages remis par la rédaction de la MBC. Ils le font à travers un outil : la schématisation. Celui-ci a tout d'abord été initié et développé par Monique à travers l'exercice particulier de la traduction en LSF du bulletin d'informations diffusé quotidiennement à France Télévisions. Elle le formalise à l'Ile Maurice pour les raisons suivantes :

- la déverbalisation des brèves à travers des représentations visuelles autour desquelles l'équipe peut échanger sur le sens et la justesse des contenus. 
- leur mémorisation par les présentatrices lors de la séance de préparation et leur calage sur l'énonciation de la journaliste à l'écran.

- $\quad$ son utilisation comme «prompteur » en pied de caméra au moment de l'enregistrement en studio.

- sa capacité à se dispenser des références aux signifiants (les signes standards) et s'intéresser principalement au signifié (sens) permettant ainsi l'émergence ou le renseignement de nouveaux signes. Le JT Hebdomadaire en MSL s'appuie ainsi essentiellement sur le «donner-à-voir en disant» des LS (structures de Grande Iconicité) défini par Christian Cuxac, linguiste à l'Université Paris 8.

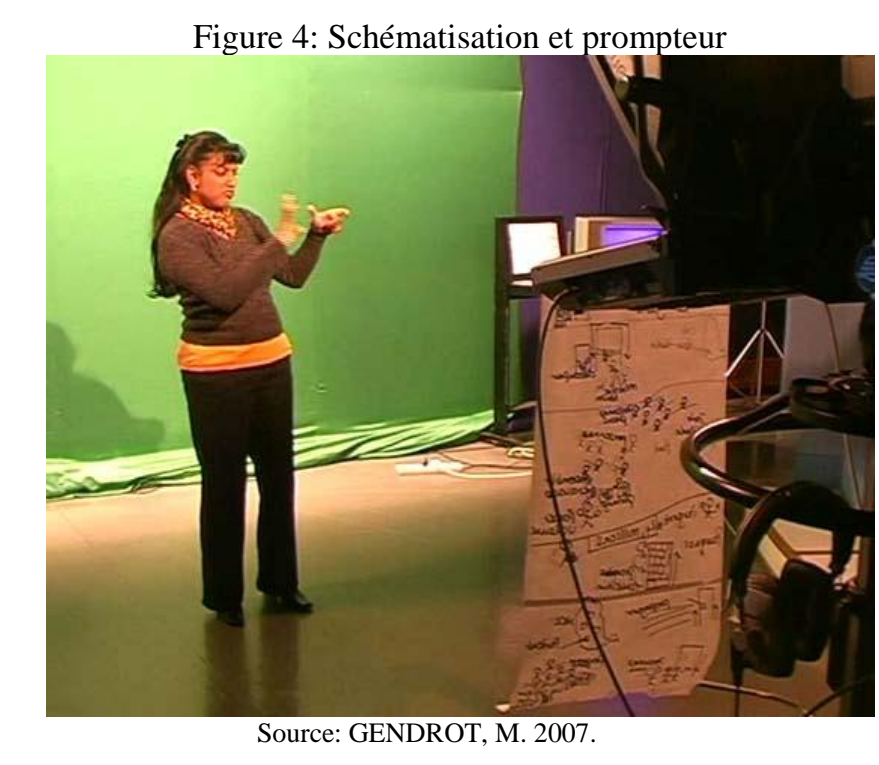

Notons des schématisations différentes selon les présentatrices. En effet, chacune possède un découpage singulier de la réalité du monde et s'y réfère. Les schématisations peuvent ainsi s'organiser sur un plan plus ou moins linéaire ou spatialisé selon leur niveau de maitrise de l'écrit. Elles peuvent aussi varier selon le sujet abordé. Pour exemple, une des présentatrices dont les schématisations se déclinent plutôt sur un mode linéaire avec le recours fréquent à l'écrit inverse totalement ses représentations (éminemment visuelles) lorsqu'elle se retrouve devant un sujet dont elle ignore le champ lexical. 
Figure 4: Schématisation de 2 brèves d'actualité
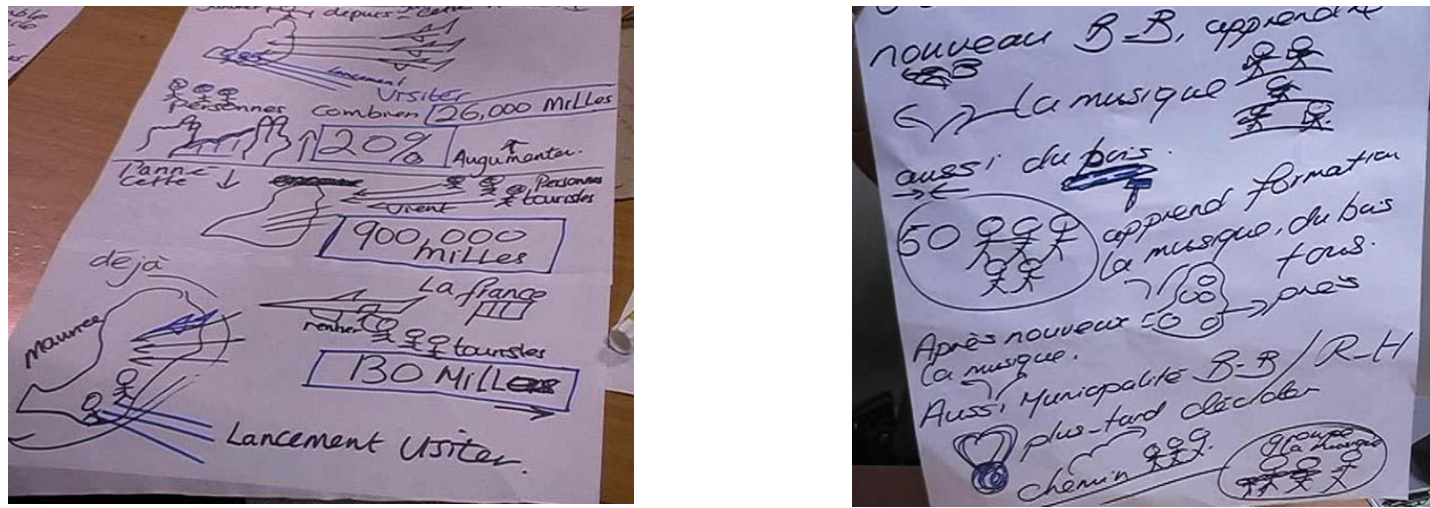

Source: GENDROT, M. 2007.

\section{Un projet similaire developpe aux Seychelles}

\subsection{Historique du Projet de Développement de la Langue des Signes Seychelloise} (SSL)

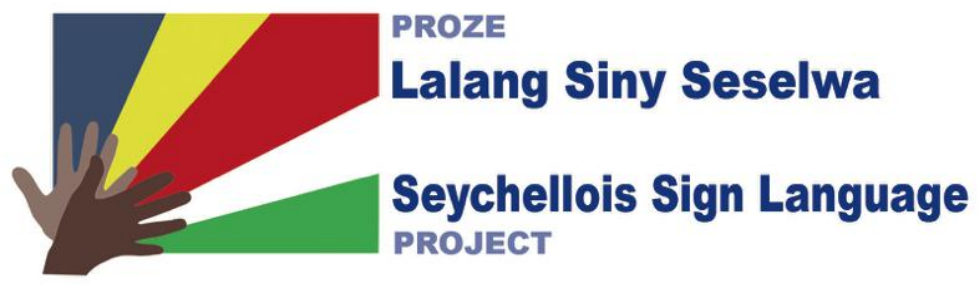

En Mai 2006, au moment de la Cérémonie de lancement du Premier Tome du Dictionnaire de MSL à l'Ile Maurice, deux ressortissantes seychelloises sont dans la salle. La première est présidente de l'Association des Sourds des Seychelles (A.P.H.I) accompagnée de son assistante. A cette occasion elles rencontrent Alain et lui demandent d'initier un projet analogue aux Seychelles. En effet, à l'instar de l'Ile Maurice, la Langue des Signes Seychelloise n'est pas encore renseignée et manque d'être reconnue. Elle a déjà subie plusieurs vagues d'influence, en particulier la LSF via des missions locales assurées par des réunionnais.

Aujourd'hui une convention est en passe d'être signée entre le gouvernement seychellois et l'INJS de Paris. Deux axes principaux s'en dégagent :

- la réalisation et la publication d'un Dictionnaire de SSL

- la formation pédagogique des futurs enseignants de SSL Sourds et Entendants 
Notons l'absence d'école spécialisée pour jeunes sourds aux Seychelles. En effet, au sein des structures, ces derniers rejoignent les autres jeunes tout handicap confondu. Ils manquent d'une prise en charge spécifique, en particulier concernant la communication et l'accès aux connaissances via la SSL. Le gouvernement seychellois conscient de l'enjeu et de l'importance d'une éducation spécifique cherche à constituer une classe d'enseignement spécialisé en attendant la construction d'une école. Les deux axes évoqués plus haut répondent à cet enjeu.

\subsection{Le Dictionnaire de SSL}

Si la méthodologie pour renseigner la langue est comparable à celle employée pour le premier tome du Dictionnaire de MSL, la réalisation de l'ouvrage connaît des modifications. En effet, les Sourds eux-mêmes s'y impliquent davantage sous l'impulsion d'Alain. Celui-ci les a formés au recueil de corpus (outil caméra), à l'extraction des signes, à leur validation au cours de séances collectives au sein de l'Association, puis à leur illustration (outils ordinateur + logiciels Photoshop) au cours de ses missions sur le terrain. Ces dernières sont au nombre de 3 , toutes programmées sur des temps de congés scolaires. La première effectuée début janvier 2007 était principalement consacrée à un «état des lieux» de la situation des Sourds aux Seychelles. Elle regroupait Alain, Monique et Nasreddine Chab, animateur Sourd du Musée des Arts et Métiers (CNAM). L'archipel des Seychelles se compose de 115 îles et un certain nombre de sourds s'y retrouvent isolés. Nous avons pu ainsi découvrir et recueillir des systèmes linguistiques développés par ces derniers au sein de leur famille, en l'absence de tout contact avec d'autres sourds et sans avoir jamais été scolarisés.

Aujourd'hui, 450 signes de SSL ont été extraits des corpus recueillis, analysés, validés et illustrés par les Sourds eux-mêmes. Notons une grande maitrise des outils et la finesse de l'illustration des signes. Ceux-ci incluent une grande richesse dans les expressions du visage et tiennent compte de l'importance du regard. La validation finale s'effectue sous la supervision d'Alain et Annie Risler, linguiste de l'Université Lille 3, avant d'être définitivement retenue pour la publication du Dictionnaire de SSL. Celle-ci devrait avoir lieu en 2017. 
Figure 5: Signes illustrés par les Sourds seychellois et extrait de la base de donnée du futur dictionnaire SSL
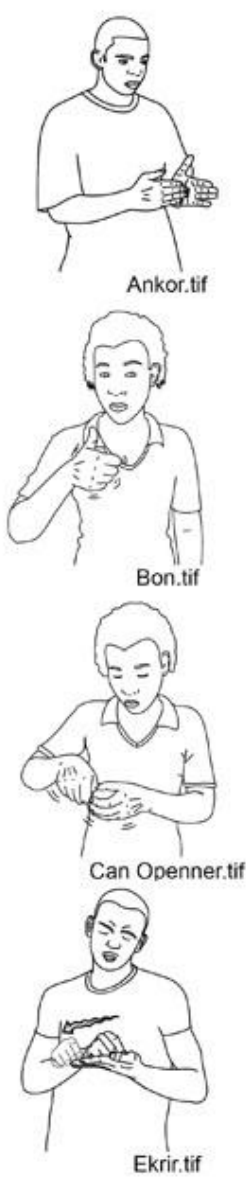
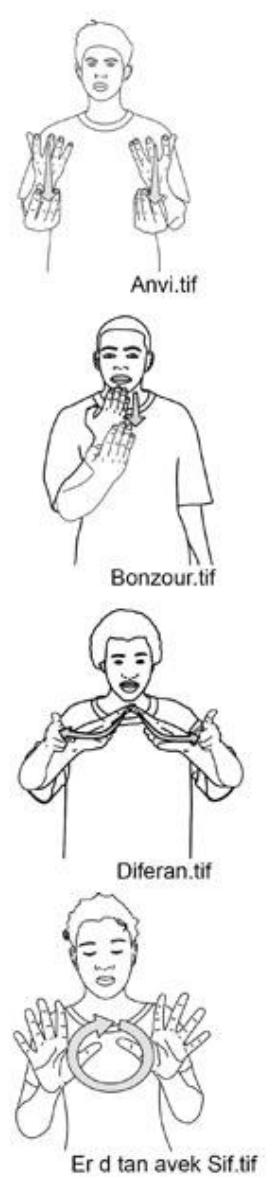
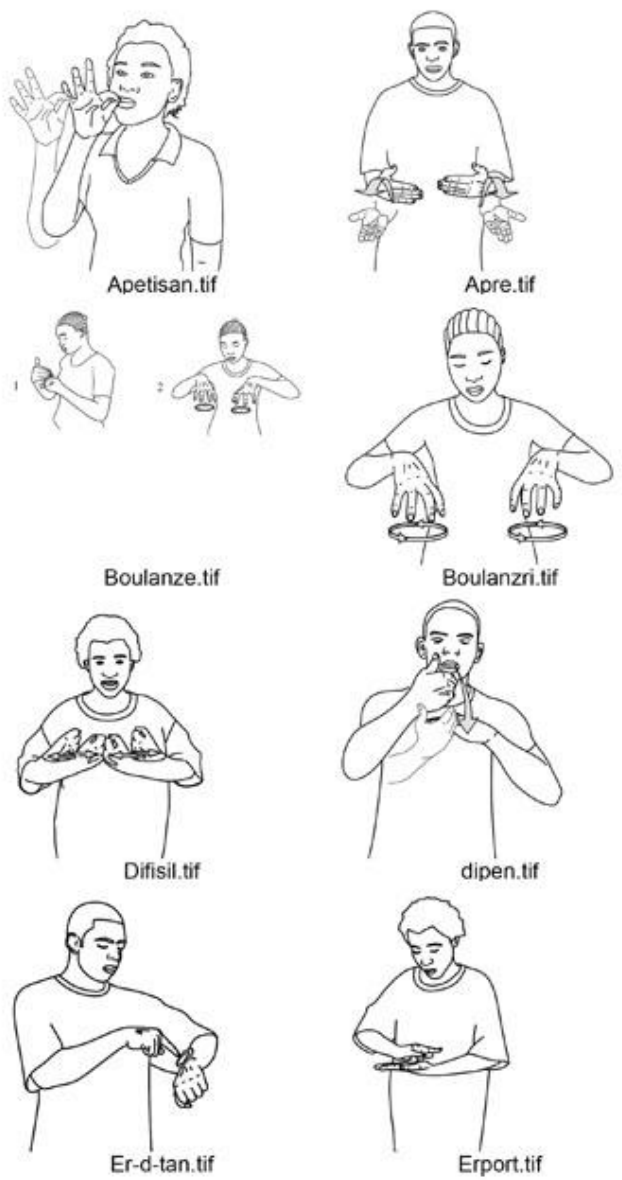
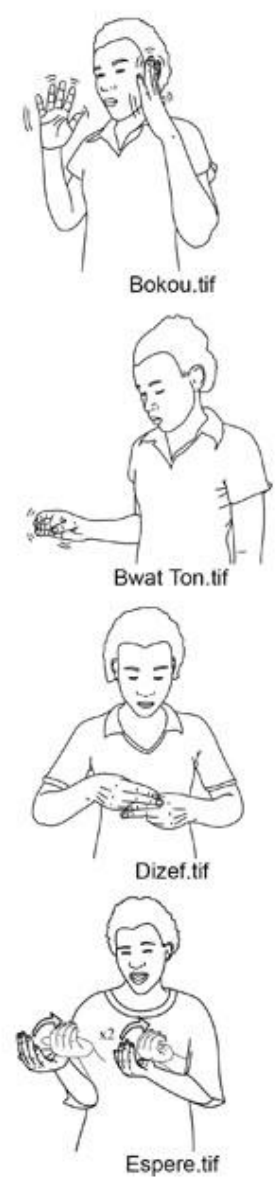

Source: GEBERT A. BOTSOIE B, 2008

\subsection{La formation pédagogique des futurs enseignants de SSL}

Elle se partage entre des actions menées sur le terrain par Alain auprès d'un public mixte sourd et entendant et des stages accomplis au sein de l'INJS de Paris. Un premier stagiaire Sourd seychellois a été accueilli sur une période de 3 semaines en Novembre 2008, suivi de 2 autres sur une période identique en Février 2010. L'une est Sourde et Vice Présidente de l'Association A.P.H.I, la seconde est Entendante et enseignante diplômée de L'Education Nationale seychelloise. Cette dernière a été formée à la Langue des Signes Seychelloise. Elle a la charge aujourd'hui d'une première classe expérimentale d'enfants sourds aux Seychelles. Pour ce faire, elle est accompagnée de sa collègue sourde, formée à la pédagogie et enseignante de SSL. 


\section{Les projets de developpement de la MSL et de la SSL : leurs retombees sur les activites du pole LSF a l'INJS de Paris}

Précisons-le d'emblée si les projets mauriciens et seychellois nous ont offert l'opportunité d'exporter et relayer nos compétences auprès de nos partenaires étrangers, ils nous ont conduits parallèlement à en acquérir de nouvelles. En effet, nous confronter à des LS émergentes et non encore renseignées, nous a appris à oublier nos «standards » pour adopter la façon de voir et de «donner-à-voir en disant » de l'Autre dans le respect de sa culture, éloignée de la nôtre. Nous plaçons ainsi cet Autre dans un double mouvement : celui auprès duquel nous apportons notre expertise et nos moyens, tout en le laissant nous apprendre tout de sa langue et de sa culture. Ce rapport particulier est un rapport d'égalité où chacun a besoin de l'autre dans ses domaines de compétences particuliers. Cette aptitude et cette capacité développées à leurs contacts nous permettent aujourd'hui d'envisager les élèves de Saint-Jacques sous le même angle. Locuteurs de leur langue, la LSF, ils sont nos informateurs et ont tout à nous apprendre de l'iconisation de leur expérience dans leur domaine de compétences qui sont les leurs au lycée professionnel. Nous recueillons ainsi des corpus de Langue des Signes auprès des élèves de plusieurs filières professionnelles au sein même de leur atelier: Métiers de la Communication et Industries Graphiques, Prothèse Dentaire, Installation Sanitaire, Serrurerie Métallerie, Production Horticole, Menuiserie, et Coiffure.

\subsection{Trois lexiques de signes LS professionnels sur le site Internet de l'INJS}

Trois lexiques de signes LS professionnels sont actuellement consultables sur le site Internet de l'INJS de Paris, avant sa refonte prévue pour la rentrée prochaine 20162017. En effet, si nous avons commencé à renseigner le lexique professionnel sous la forme de glossaires, c'est-à-dire un terme lexical en français et sa correspondance en langue des signes, nous l'avons poursuivi en l'enrichissant de «définitions » dans les deux langues français/langue des signes. Ce travail est publié et visible à la rubrique pour trois des filières professionnelles: http://www.injs-paris.fr/plateforme-lexiquefrancais-lsf 
Aujourd'hui, il s'agit de poursuivre le renseignement des autres filières en les publiant sur le modèle d'un dictionnaire numérique spécialisé des métiers conçu par Alain. C'est pourquoi notre direction devant l'intérêt d'un tel projet le soutient en optant pour une refonte du site.

Toutefois, le recueil des signes auprès des jeunes des classes de terminale, accompagnés de leur professeur, s'effectue toujours selon les méthodes initiées au cours des projets mauricien et seychellois. Il répond aux objectifs suivants :

- renseigner la langue dans des domaines de spécificité où le lexique standard tend à manquer

- constituer un lexique en contexte en recueillant les néologismes mis en place par les élèves à travers l'iconisation de leur expérience d'apprentis.

- valider les signes extraits en présence des élèves pour leur versant LS et des professeurs pour leur terminologie technique en français.

- diffuser les signes validés à l'ensemble des classes de la filière présentes et à venir.

Figure 6: Fiche de saisie des entrées des Unités Lexicales et de leur description

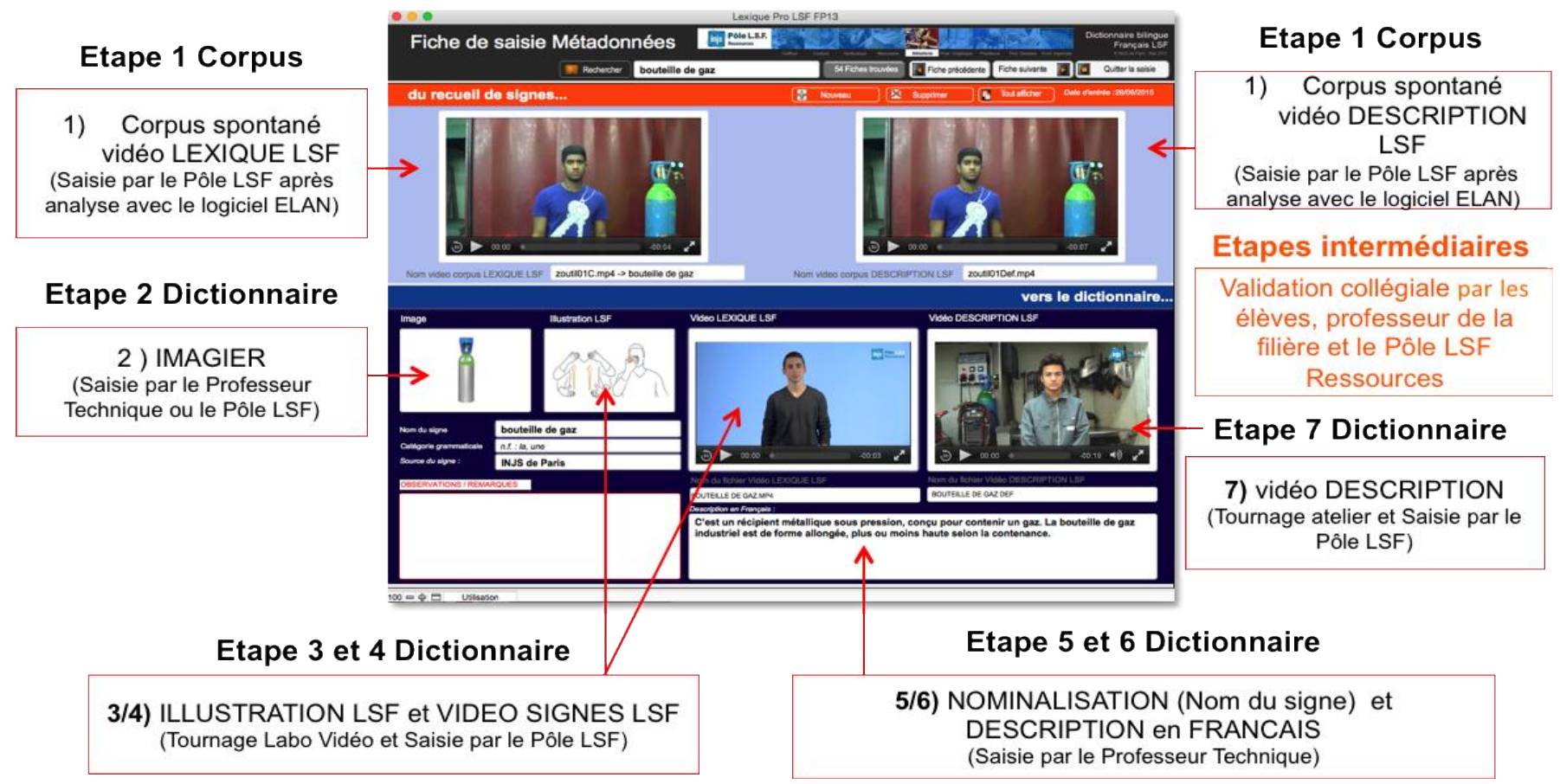

Source: GEBERT, A., 2015. 
Figure 7: Intégration future du modèle dans le site internet de l'INJS

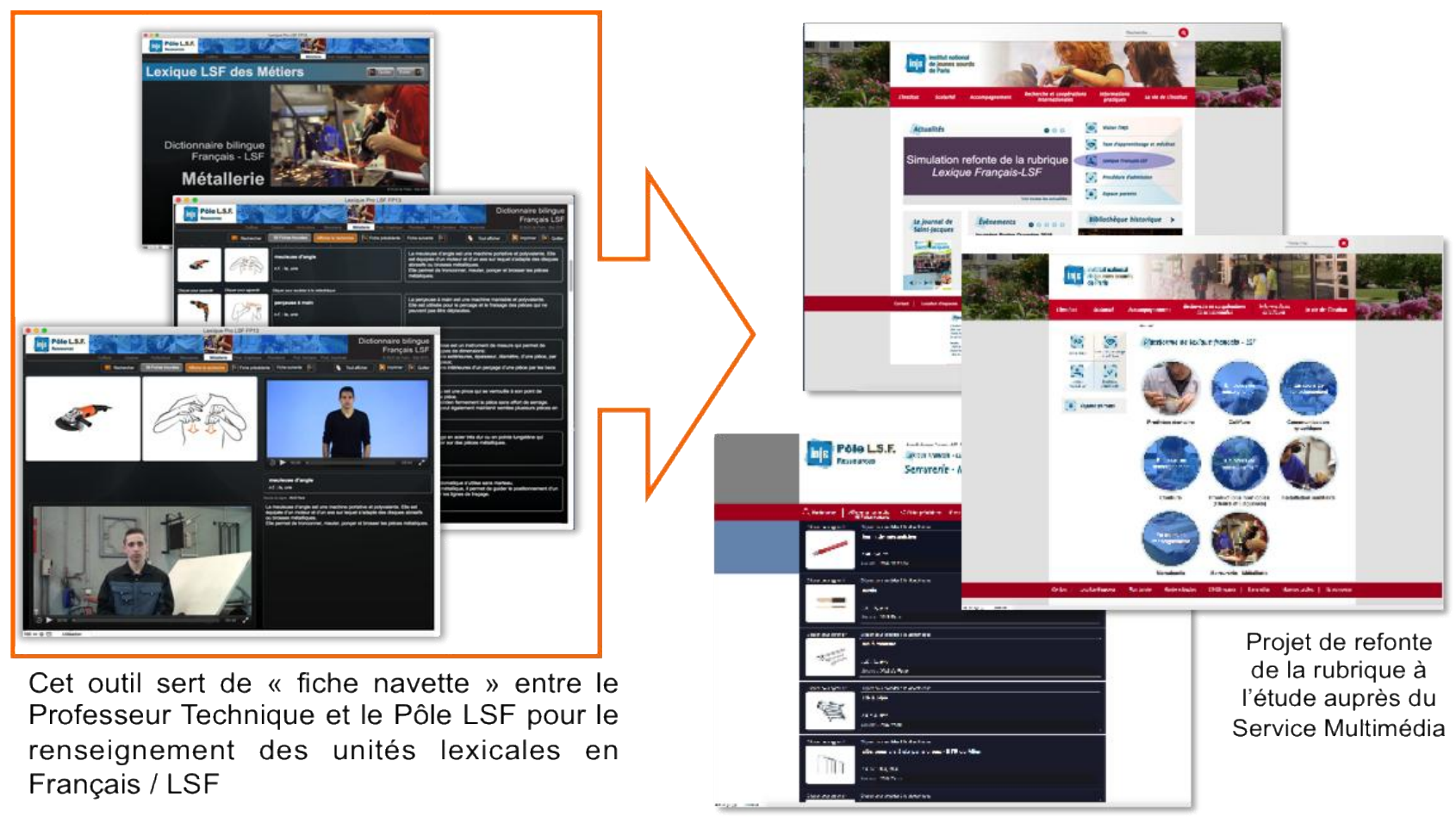

Source: GEBERT, A., 2015.

Au-delà des objectifs lexicaux, cette activité présente l'avantage de :

- mettre les jeunes au centre des activités du Pôle

- favoriser le changement de regard des jeunes sur eux-mêmes, locuteurs et informateurs de LS, sujet d'intérêt et d'étude pour eux-mêmes et les autres

- participer au changement de regard des professeurs sur la LS et ses capacités de «donner-à-voir en disant» au moment où le vocabulaire standard est absent et les néologismes se mettent en place

- participer à l'acquisition des procédures ou l'appropriation des connaissances par les élèves en présence de leurs professeurs au cours des séances de validation collective. En effet, certaines notions sont alors complétées ou bien précisées.

\subsection{La schématisation}

Cet outil formalisé par Monique à l'Ile Maurice est aujourd'hui utilisé dans les classes d'enseignement de la LSF et en LSF par les professeurs du Collège et Lycée de l'INJS. En effet, il répond à une transcription visuelle des concepts et des signifiés. Il 
participe de la construction d'un énoncé en LSF et à sa mémorisation. Il est également proposé aux professeurs des matières d'enseignement général qui souhaite s'y initier.

Figure 8: Exemple de mise en application de la Schématisation dans un cours de LSF en classe de $4^{\text {ème }}$

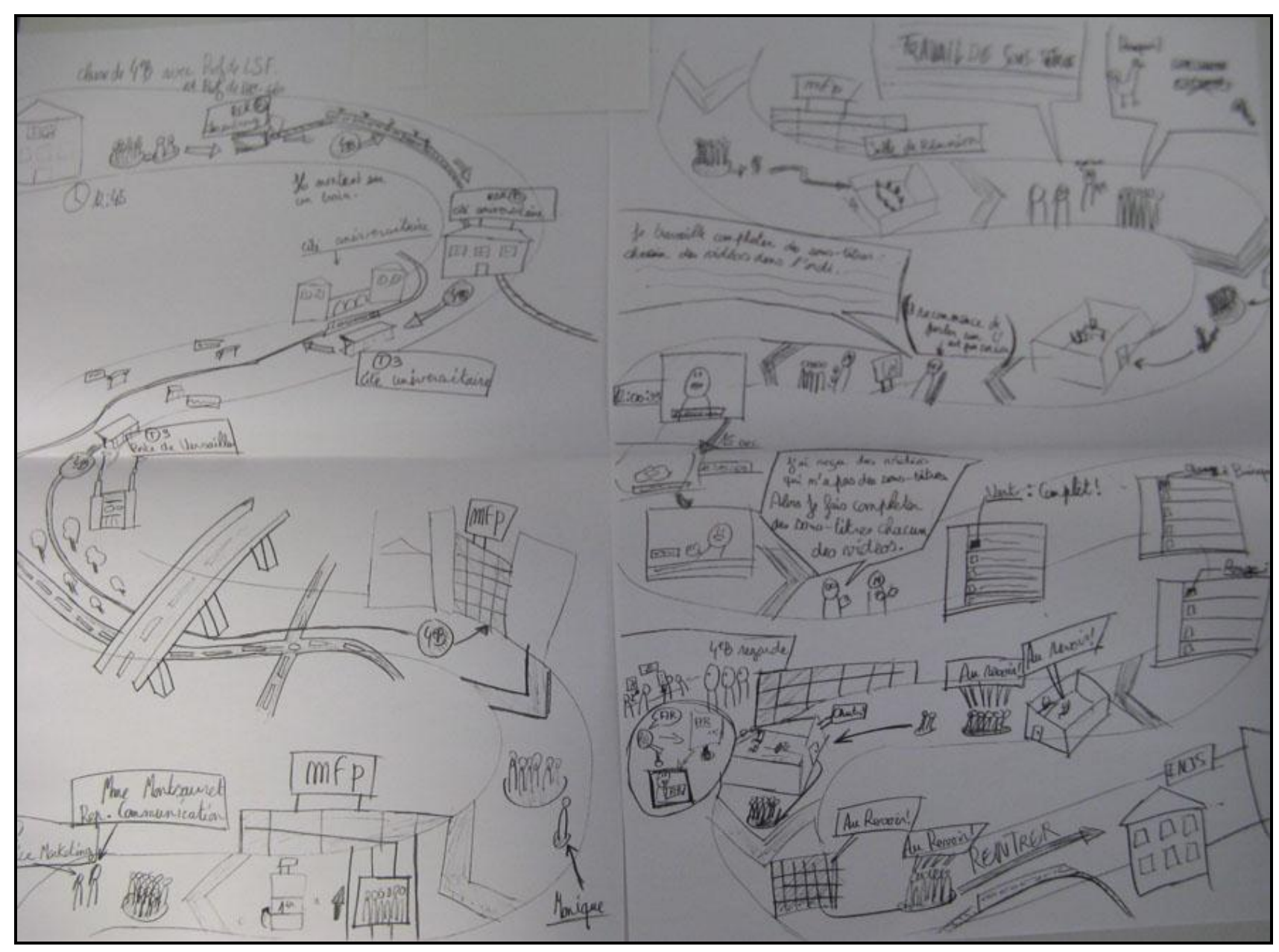

Source: VENUTTI, A., 2009.

\section{Conclusion}

Les projets et actions présentés dans cet article ont faits l'objet de plusieurs communications dans diverses conférences internationales (Universités de Preston et de Leiden, Institut National de Jeunes Sourds à Rio de Janeiro..). A chaque fois nous y avons redécouvert le caractère anthropologique de nos travaux accomplis sur le terrain et au plus près des langues renseignées dans le contexte même d'où elles émergent. En 
effet, plusieurs fois, en particulier, auprès des sourds isolés développant leur propre système linguistique nous avons eu le sentiment de pénétrer au cœur de l'humanité.

\section{RÉFÉRENCE}

CUXAC, C. La Langue des Signes Française ; les Voies de l'Iconicité; Faits de langues, $\mathrm{N}^{\circ} 15-16$, Ophrys, Paris, 2000.

BREZINGER, M., BATIBO, H., Atlas des Langues en danger dans le monde ; p. 27 ; «Les Langues des signes africaines en danger ». Editions UNESCO ; 2010.

GENDROT, M., Avenant au mémoire de recherche. L'interprète en institution : à la croisée des regards »; Master 2 Interprétation Français/LSF ; Université Paris 8 ; 2008.

GEBERT, A.; LUCBERNET. C.; TOKHTCHOUKOV. A.; mémoire de Licence Professionnelle Enseignement de la Langue des Signes Française (LSF) en milieu scolaire ; Le développement de la pensée visuelle chez l'apprenant entendant dans un cours de LSF; Université de Paris 8 ; 2007.

.; Rapport de stage; Master 1ère année Mention Sciences du langage Spécialité Lexicographie, Terminographie, et Traitement Automatique des Corpus (LTTAC); Conception d'un dictionnaire numérique professionnel bilingue français/LSF de la filière Métallerie; Université de Lille 3 ; 2015. 